Ciência Florestal, Santa Maria, v. 20, n. 4, p. 665-674, out.-dez., 2010

\title{
DENSIDADE BÁSICA E RETRATIBILIDADE DA MADEIRA DE CLONES DE TRÊS ESPÉCIES DE Eucalyptus
}

\author{
BASIC DENSITY AND RETRACTIBILITY OF WOOD CLONES OF THREE \\ Eucalyptus SPECIES
}

\author{
Djeison Cesar Batista ${ }^{1}$ Ricardo Jorge Klitzke ${ }^{2}$ Carlos Vinícius Taborda Santos ${ }^{3}$
}

\begin{abstract}
RESUMO
Dentre as florestas plantadas que abastecem a indústria madeireira nacional, as do gênero Eucalyptus estão entre as mais importantes. Essa importância é atribuída à velocidade de desenvolvimento de suas árvores, facilidade de implantação em grandes maciços e versatilidade de aplicação de sua madeira. A criação de híbridos e clones é uma realidade na silvicultura nacional, na qual o melhoramento genético busca sobretudo maiores incrementos volumétricos e resistência a condições extremas de implantação, tais como ataques de pragas, secas, geadas e baixa fertilidade do solo. A densidade básica é uma das propriedades físicas mais importantes da madeira por se relacionar diretamente com outras propriedades da madeira, inclusive a anisotropia de contração. Assim, tais propriedades da madeira norteiam a utilização racional de uma espécie em um determinado produto. O objetivo deste trabalho foi determinar a densidade básica e a anisotropia de contração da madeira de cinco clones das espécies Eucalyptus saligna, Eucalyptus grandis e Eucalyptus dunnii. O clone 5 de Eucalyptus saligna obteve a maior densidade básica $\left(0,56 \mathrm{~g} / \mathrm{cm}^{3}\right)$ e foi o mais instável dimensionalmente. Dentre todas as espécies, observou-se relação direta entre densidade básica e contração volumétrica máxima e entre densidade básica e coeficiente de retratibilidade volumétrico máximo somente para o clone 5 de Eucalyptus saligna. Considerando-se como critério a contração volumétrica máxima, o clone 3 foi o mais estável dimensionalmente. Para o Eucalyptus grandis, os clones 2 e 3 obtiveram, respectivamente, a menor e maior densidade básica, com 0,40 e $0,49 \mathrm{~g} / \mathrm{cm}^{3}$. Não foi possível distinguir dentre os clones 1,3 e 4 qual foi o mais estável dimensionalmente e, considerando-se como critério o coeficiente de retratibilidade volumétrico máximo, o clone 5 foi o mais instável dimensionalmente. Para as espécies Eucalyptus saligna e Eucalyptus dunnii não foi possível concluir qual clone obteve a menor densidade básica. O clone 3 de Eucalyptus dunnii obteve a maior densidade básica $\left(0,65 \mathrm{~g} / \mathrm{cm}^{3}\right)$ e, considerando-se como critério o coeficiente de retratibilidade volumétrico máximo, foi o clone mais instável dimensionalmente. Considerando-se a contração volumétrica máxima, o clone 1 foi o mais estável dimensionalmente. O Eucalyptus grandis foi a espécie de menor densidade e maior estabilidade dimensional, enquanto o Eucalyptus dunnii foi a espécie de maior densidade e maior instabilidade dimensional.
\end{abstract}

Palavras-chave: clones; madeira; densidade básica; anisotropia de contração.

\begin{abstract}
Among the planted forests that supply the national wood industry, the genus Eucalyptus has become the most important, due to its fast growth, ease of large scale planting and variability of wood use. The generation of new hybrids and clones is a reality in the national practice of silviculture, and there is great interest currently in finding genetic improvements, mainly for higher volumetric gains and resistance in rough conditions of planting, such as pest attacks, periods of drought, low soil fertility, etc. The basic density is one of the

1. Engenheiro Florestal, M.Sc., Professor Assistente do Departamento de Engenharia Florestal, Centro de Ciências Agrárias, Universidade Federal do Espírito Santo, Av. Governador Carlos Lindemberg, 316, Bairro Centro, CEP 29550-000, Jerônimo Monteiro (ES). djeison1984@gmail.com

2. Engenheiro Florestal, Dr., Professor Adjunto do Departamento de Engenharia e Tecnologia Florestal, Setor de Ciências Agrárias, Centro de Ciências Florestais e da Madeira, Universidade Federal do Paraná, Campus III, Av. Lothario Meissner, 900, Jardim Botânico,CEP 80210-170, Curitiba (PR).rklitzke@ufpr.br

3. Engenheiro Industrial Madeireiro, Departamento de Engenharia e Tecnologia Florestal, Setor de Ciências Agrárias, Centro de Ciências Florestais e da Madeira, Universidade Federal do Paraná, Campus III, Av. Lothario Meissner, 900, Jardim Botânico, CEP 80210-170, Curitiba (PR). carlostabordamadeira@yahoo.com.br

Recebido para publicação em 12/03/2009 e aceito em 2/07/2010
\end{abstract}

Ci. Fl., v. 20, n. 4, out.-dez., 2010 
most important physical properties of wood because it relates directly to other properties, including the anisotropic shrinkage. Such properties indicate the rational use of a species in a certain wood product. The aim of this work was to determine the basic density and the anisotropic shrinkage of five wood clones for each one of the following species: Eucalyptus saligna, Eucalyptus grandis and Eucalyptus dunnii. Clone 5 of Eucalyptus saligna presented the highest basic density $\left(0.56 \mathrm{~g} / \mathrm{cm}^{3}\right)$ and was the most dimensionally instable. Of all the species, there was only a direct relation among basic density, maximum volumetric shrinkage and maximum volumetric shrinkage coefficient in this clone. Considering maximum volumetric shrinkage as the criterion, clone 3 was the most dimensionally stable. Clones 2 and 3 of Eucalyptus grandis presented the least and the highest basic density, respectively, with 0.40 and $0.49 \mathrm{~g} / \mathrm{cm}^{3}$. It was not possible to distinguish among clones 1, 3 and 4 in terms of dimensional stability, and considering maximum volumetric shrinkage coefficient as the criterion, clone 5 was the most dimensionally instable. For Eucalyptus saligna and Eucalyptus dunnii it was not possible to distinguish which clone presented the least basic density. Clone 3 of Eucalyptus dunnii presented the highest basic density $\left(0.65 \mathrm{~g} / \mathrm{cm}^{3}\right)$ and considering maximum volumetric shrinkage coefficient as the criterion, it was the most dimensionally instable clone, whereas considering maximum volumetric shrinkage, clone 1 was the most stable. Eucalyptus grandis presented the least basic density and was the most stable, while Eucalyptus dunnii presented the highest basic density and was the most dimensionally instable.

Keywords: wood; clones; basic density; anisotropy of shrinkage.

\section{INTRODUÇÃO}

Dentre as florestas plantadas que abastecem a indústria madeireira nacional, as do gênero Eucalyptus estão entre as mais importantes. Essa importância é atribuída à velocidade de desenvolvimento de suas árvores, facilidade de implantação em grandes maciços e versatilidade de aplicação de sua madeira

Para a utilização industrial é desejável que a madeira, enquanto matéria-prima, tenha uniformidade nas características de cor, densidade básica, resistência mecânica e trabalhabilidade. No entanto, é conhecido que a madeira é um material heterogêneo, o que dificulta o atendimento de todas essas características desejáveis. Porém, os plantios clonais de Eucalyptus têm possibilidade de produzir madeira mais homogênea, o que pode refletir em maior qualidade no produto final.

$\mathrm{O}$ potencial da aplicação da madeira de Eucalyptus é em razão de sua diversidade de espécies, alta capacidade de geração de clones e híbridos. Essa diversidade permite a introdução do gênero em programas de melhoramento genético, de condução da floresta, de manejo e uso de tecnologias adequadas de processamento e usinagem, que se mostrem eficientes na solução ou diminuição de defeitos, tais como as rachaduras de topo e empenamentos, em toras e tábuas (TRUGILHO et al., 2006).

De acordo com informações dos Anais da I Reunião Regional Sobre Clonagem Intensiva de Eucalyptus (1994), já àquela época, a utilização de híbridos em escala comercial era um aspecto que vinha despertando a atenção dos silvicultores nacionais, e que a clonagem tornara-se prática rotineira nos programas florestais das principais empresas nacionais, sendo os principais responsáveis pelo sucesso da eucaliptocultura nacional. Os pesquisadores já alertavam para o fato de que, apesar dos clones apresentarem alta produtividade volumétrica em madeira, seriam necessários maiores estudos quanto à sua qualidade.

A estrutura anatômica influencia diretamente na densidade da madeira, e esta última, por sua vez, também exerce influência direta na anisotropia. Portanto, trata-se de propriedades estreitamente correlatas. Kollmann e Côté Júnior (1968) afirmam que quanto maior a densidade da madeira, maiores são a contração e o inchamento volumétricos, havendo uma relação praticamente linear entre essas propriedades.

Klitzke (2007) relacionou que, dentre os fatores inerentes à própria espécie de madeira que influenciam na sua secagem, encontram-se a estrutura anatômica, a densidade e a anisotropia. Esse autor ainda corrobora que a mudança de volume verificada na madeira em consequência da desorção e adsorção de água é considerada umas das propriedades físicas mais importantes, afetando e limitando a utilização industrial.

A densidade é definida fisicamente como "massa de amostra por unidade de volume". Por coincidência de definições, a grandeza física de massa do Sistema Internacional de Unidades (SI) é 
praticamente igual ao valor numérico de peso, em unidades do sistema MKS (MELO, 2002).

Dentre as diversas propriedades da madeira, a densidade é a mais utilizada, pela facilidade de ser determinada e por se correlacionar diretamente com as propriedades físicas e mecânicas da madeira e com a composição celular.

Andrade, Jankowsky e Ducattii (2001) obtiveram densidade básica de $0,54 \mathrm{~g} / \mathrm{cm}^{3}$ para a madeira de Eucalyptus grandis, enquanto Ciniglio (1998) obteve densidade básica de $0,57 \mathrm{~g} / \mathrm{cm}^{3}$ para a idade de 18 anos. Pereira et al. (2000) estudaram árvores de Eucalyptus grandis de 6, 8, 9 e 10,5 anos de idade e obtiveram valores de densidade básica que variaram de $0,36 \mathrm{~g} / \mathrm{cm}^{3}$ a $0,51 \mathrm{~g} / \mathrm{cm}^{3}$. Tomazello Filho (1985) obteve densidade básica média de 0,38 $\mathrm{g} / \mathrm{cm}^{3}$ para árvores de Eucalyptus grandis aos 10 anos de idade.

Trugilho, Lima e Mendes (1996) estudaram as características da madeira juvenil de Eucalyptus saligna e obtiveram densidades básicas de até 0,52 $\mathrm{g} / \mathrm{cm}^{3}$. Pereira et al. (2000) estudaram plantios de Eucalyptus saligna e obtiveram valores de densidade básica de $0,44 \mathrm{~g} / \mathrm{cm}^{3}, 0,53 \mathrm{~g} / \mathrm{cm}^{3}$ e $0,55 \mathrm{~g} / \mathrm{cm}^{3}$ para as idades de 9,8 e 10,5 anos respectivamente. Tomazello Filho (1985) estudou duas procedências de Eucalyptus saligna aos 10 anos de idade e obteve densidades básicas médias de $0,43 \mathrm{~g} / \mathrm{cm}^{3}$ e $0,50 \mathrm{~g} /$ $\mathrm{cm}^{3}$.

Pereira et al. (2000) obtiveram $0,55 \mathrm{~g} / \mathrm{cm}^{3}$ de densidade básica média para árvores de 8 anos de Eucalyptus dunnii, enquanto Severo (1998) obteve densidade média de $0,57 \mathrm{~g} / \mathrm{cm}^{3}$ e $0,58 \mathrm{~g} / \mathrm{cm}^{3}$ para duas procedências de 16 anos. Rocha (2000) obteve densidade básica média de $0,62 \mathrm{~g} / \mathrm{cm}^{3}$ para árvores de Eucalyptus dunnii de 16,5 anos de idade.

O material madeira possui outra característica física muito importante do ponto de vista da sua aplicação, chamada anisotropia, que é definida como um comportamento desigual nas diferentes direções estruturais da madeira - longitudinal, tangencial e radial. Esta característica torna a madeira um material peculiar, diferenciando-a dos metais e plásticos. A maior variação dimensional ocorre na direção tangencial aos anéis de crescimento, e a contração radial é menor do que a tangencial. A contração tangencial da madeira desde o estado verde (acima do ponto de saturação das fibras) até seco em estufa ( $0 \%$ de umidade) encontra-se no intervalo de $3,5 \%$ a $15,0 \%$, enquanto a contração radial encontra-se na faixa de 2,4\% a 11,0\% (KOLLMANN E CÔTÉ JÚNIOR,
1968).

Gonçalez et al. (2006) obtiveram contração máxima volumétrica, radial e tangencial de respectivamente $17,3 \%, 6,9 \%$ e $10,9 \%$ para a madeira de Eucalyptus grandis de 17 anos de idade e anisotropia de contração de 1,58. Silva et al. (2006) estudaram diferentes idades e posições do tronco de Eucalyptus grandis e obtiveram contrações máximas volumétrica, radial e tangencial respectivas de 18,1, 6,1 e $10,1 \%$ e anisotropia de contração de 1,71 .

Oliveira e Silva (2003) estudaram a madeira de Eucalyptus saligna de 16 anos de idade e obtiveram contração máxima volumétrica, radial e tangencial de, respectivamente, 26,0, 7,7 e $14,8 \%$ e anisotropia de contração de 1,99. Malan (2000) apud Marchesan, Mattos e Shimizu (2005) obtiveram contração máxima volumétrica, radial e tangencial de, respectivamente, 16,3, 6,3 e 9,8\% e anisotropia de contração de 1,56.

Rocha (2000) obteve contração máxima volumétrica, radial e tangencial de, respectivamente, $19,2,7,9$ e 12,1\% para madeira de Eucalyptus dunnii de 16,5 anos de idade. A anisotropia de contração obtida por este autor foi de 1,55. Severo (1998) estudou dois tratamentos para duas procedências de Eucalyptus dunnii e obteve valores de contração volumétrica máxima de 20,8 e 23,5\% para a madeira da procedência Urbenville e de 20,5 e $24,4 \%$ para a procedência Dorrigo.

O objetivo deste trabalho foi determinar a densidade básica e a anisotropia de contração da madeira de cinco clones das espécies Eucalyptus saligna, Eucalyptus grandis e Eucalyptus dunnii.

\section{MATERIAL E MÉTODOS}

\section{Caracterização do Material}

As árvores foram coletadas de plantios clonais homogêneos de 11 anos de idade, propagados por estacas, plantadas em espaçamento 2,5 x 3,0 $\mathrm{m}$ na cidade de Telêmaco Borba, região centro oriental do estado do Paraná. Neste estudo, foram utilizados 15 diferentes clones, cinco de cada uma das espécies a seguir: Eucalyptus saligna Smith, Eucalyptus grandis W.Hill ex Maiden e Eucalyptus dunnii Maiden.

\section{Amostragem}

De cada clone foram coletadas cinco árvores de fuste retilíneo e aparentemente sadias. Apenas 
árvores de diâmetro à altura do peito superior a 20 $\mathrm{cm}$ foram escolhidas. Cada árvore foi derrubada com motosserra, a $10 \mathrm{~cm}$ do solo, utilizando-se apenas a primeira tora, de comprimento igual a $3 \mathrm{~m}$. Em seguida, as toras foram levadas para serraria onde foram desdobradas em tábuas. De cada árvore, utilizaram-se duas tábuas tangenciais livres de defeitos (nós, esmoado, rachaduras, bolsas de goma, etc.), localizadas na porção intermediária do diâmetro, ou seja, entre a medula e a casca, de acordo com a Figura 1. Dessa maneira, utilizaramse dez tábuas por clone.

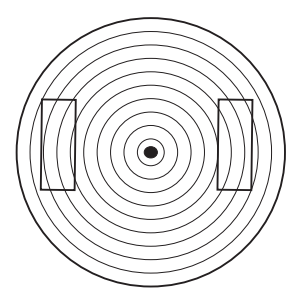

FIGURA 1: Esquema de amostragem das tábuas. FIGURE 1: Board sampling scheme.

De cada tábua foram confeccionados cinco corpos-de-prova devidamente orientados, de dimensões 25 × 25 x $100 \mathrm{~mm}$, utilizando-se apenas a porção média destas, de acordo com a Figura 2. Dessa maneira, utilizaram-se dez corpos-de-prova por árvore, totalizando cinquenta corpos-de-prova por clone.

\section{Ensaio de densidade e anisotropia de contração}

Neste trabalho, foram determinadas a densidade básica e a anisotropia de contração

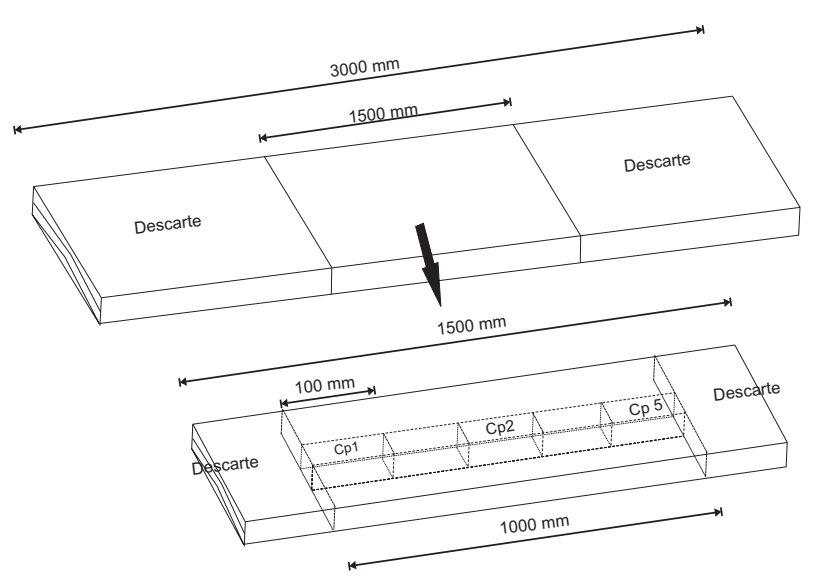

FIGURA 2: Porção das tábuas utilizada na confecção dos corpos-de-prova.

FIGURE 2: Part of the boards used for sampling specimens. da madeira dos clones, de acordo com as normas COPANT 461 e 462 (1972) respectivamente. O ensaio de densidade básica foi modificado no tocante à dimensão dos corpos-de-prova, utilizando-se os mesmos corpos-de-prova do ensaio de anisotropia de contração. Isso ocorreu em conseqüência da limitação da espessura média das tábuas, que foi de $30 \mathrm{~mm}$

No ensaio de anisotropia de contração, obtiveram-se a contração máxima volumétrica, radial e tangencial, o coeficiente de anisotropia de contração, além dos coeficientes de retratibilidade volumétrico, radial e tangencial.

A densidade básica foi calculada de acordo com a Equação 1.

$$
D B=\frac{M S}{V V}
$$

Em que:

DB: densidade básica $\left(\mathrm{g} / \mathrm{cm}^{3}\right)$;

MS: massa seca a $0 \%(\mathrm{~g})$

$\mathrm{VV}$ : volume verde $\left(\mathrm{cm}^{3}\right)$.

A contração máxima foi calculada de acordo com a Equação 2.

$$
\beta=\frac{(D V-D S)}{D V} \times 100
$$

Em que:

$\beta$ : contração máxima, em uma dada direção estrutural (\%);

DV: dimensão verde, em uma dada direção estrutural (mm);

DS: dimensão seca, em uma dada direção estrutural (mm).

O coeficiente de retratibilidade foi calculado de acordo com a Equação 3. Os valores obtidos a $12 \%$ de umidade se referem à madeira estabilizada em câmara climática a $20^{\circ} \mathrm{C}$ e $65 \%$ de umidade relativa.

$$
Q \beta=\frac{(D 12-D 0) \times M 0}{(M 12-M 0) \times D 12} \times 100
$$

Em que:

$\mathrm{Q} \beta$ : coeficiente de retratibilidade, em uma dada direção estrutural (\%/\%);

D12: dimensão a $12 \%$ de umidade, em uma dada direção estrutural $(\mathrm{mm})$;

D0: dimensão a $0 \%$ de umidade, em uma dada direção estrutural ( $\mathrm{mm})$;

M12: massa a $12 \%$ de umidade (g);

M0: massa seca a $0 \%$ de umidade.

Ci. Fl., v. 20, n. 4, out.-dez., 2010 


\section{Análise Estatística}

Para a análise dos resultados, utilizou-se a análise de variância (teste $\mathrm{F}$ a $5 \%$ de significância), e nos casos em que a hipótese da nulidade foi rejeitada, aplicou-se o teste de Tukey $(5 \%$ de significância) para a diferenciação das médias.

Para analisar o grau de associação entre a densidade básica e a contração volumétrica máxima, aplicou-se o coeficiente de correlação de Pearson.

\section{RESULTADOS E DISCUSSÃO}

\section{Densidade básica}

Na Tabela 1, encontram-se as médias de densidade básica dos clones por espécie.

A média geral da densidade do Eucalyptus saligna, de $0,46 \mathrm{~g} / \mathrm{cm}^{3}$ encontra-se dentro do intervalo proposto por Pereira et al. (2000), que estudaram árvores com as idades entre 8 e 10,5 anos de idade. No entanto, o clone 5, que apresentou a maior densidade básica média de $0,56 \mathrm{~g} / \mathrm{cm}^{3}$, foi superior aos valores relatados por esses autores. $\mathrm{O}$ clone 3 apresentou a menor densidade básica média absoluta, com $0,42 \mathrm{~g} / \mathrm{cm}^{3}$, porém não diferiu estatisticamente dos clones 4 e $2, \operatorname{com} 0,43 \mathrm{~g} / \mathrm{cm}^{3} \mathrm{e}$

TABELA 1: Densidade básica média por clone e por espécie.

TABLE 1: Average basic density of clones and species.

\begin{tabular}{cccc}
\hline \multirow{2}{*}{ Clone } & \multicolumn{3}{c}{ Densidade Básica $\left(\mathrm{g} / \mathrm{cm}^{3}\right)$} \\
\cline { 2 - 4 } & $\begin{array}{c}\text { Eucalyptus } \\
\text { saligna }\end{array}$ & $\begin{array}{c}\text { Eucalyptus } \\
\text { grandis }\end{array}$ & $\begin{array}{c}\text { Eucalyptus } \\
\text { dunnii }\end{array}$ \\
\hline \multirow{2}{*}{1} & $0,46 \mathrm{~B}$ & $0,46 \mathrm{~B}$ & $0,51 \mathrm{~A}$ \\
& $(14,1)$ & $(10,2)$ & $(9,1)$ \\
2 & $0,45 \mathrm{AB}$ & $0,49 \mathrm{C}$ & $0,51 \mathrm{~A}$ \\
& $(13,8)$ & $(7,5)$ & $(12,0)$ \\
3 & $0,42 \mathrm{~A}$ & $0,40 \mathrm{~A}$ & $0,65 \mathrm{C}$ \\
& $(12,1)$ & $(7,8)$ & $(11,8)$ \\
\multirow{2}{*}{4} & $0,43 \mathrm{AB}$ & $0,45 \mathrm{~B}$ & $0,59 \mathrm{~B}$ \\
& $(10,9)$ & $(8,5)$ & $(17,6)$ \\
5 & $0,56 \mathrm{C}$ & $0,46 \mathrm{~B}$ & $0,53 \mathrm{~A}$ \\
& $(15,5)$ & $(16,7)$ & $(13,2)$ \\
\hline \multirow{2}{*}{ Espécie } & $0,46 \mathrm{a}$ & $0,45 \mathrm{a}$ & $0,56 \mathrm{~b}$ \\
& $(13,3)$ & $(10,1)$ & $(12,7)$ \\
\hline
\end{tabular}

Médias seguidas por uma mesma letra maiúscula em uma mesma coluna e por uma mesma letra minúscula em uma mesma linha não diferem entre si de acordo com o teste de Tukey a 5\% de significância. Resultados entre parênteses correspondem ao coeficiente de variação (\%).
$0,45 \mathrm{~g} / \mathrm{cm}^{3}$ respectivamente.

A densidade básica média do Eucalyptus grandis foi de $0,45 \mathrm{~g} / \mathrm{cm}^{3}$, superior aos $0,42 \mathrm{~g} /$ $\mathrm{cm}^{3}$ obtidos por IPT (1989) apud IPT (2009), porém encontra-se dentro do intervalo proposto por Gonçalez et al. (2006) e Silva et al. (2006), que também estudaram a madeira dessa espécie.

De acordo com os resultados obtidos, o clone 2 apresentou a maior densidade básica média, com $0,49 \mathrm{~g} / \mathrm{cm}^{3}$, enquanto o clone 3 apresentou a menor densidade básica média, com $0,40 \mathrm{~g} / \mathrm{cm}^{3}$

A densidade básica média do Eucalyptus dunnii foi de $0,56 \mathrm{~g} / \mathrm{cm}^{3}$ e encontra-se de acordo com os resultados obtidos por Severo (1998) e Pereira et al. (2000). No entanto, o clone 3 obteve a maior densidade básica média, de $0,65 \mathrm{~g} / \mathrm{cm}^{3}$, superior à média de $0,62 \mathrm{~g} / \mathrm{cm}^{3}$ obtida por Rocha (2000), que trabalhou com árvores de 16,5 anos de idade. Os clones 1 e 2 apresentaram a menor densidade básica média absoluta, com $0,51 \mathrm{~g} / \mathrm{cm}^{3}$, porém não diferiram estatisticamente do clone 5 com $0,53 \mathrm{~g} /$ $\mathrm{cm}^{3}$.

O Eucalyptus dunnii foi a espécie que obteve a maior densidade básica média, com 0,56 $\mathrm{g} / \mathrm{cm}^{3}$. As médias da densidade básica das espécies Eucalyptus saligna e Eucalyptus grandis não diferiram estatisticamente.

Os coeficientes de variação da densidade básica por espécie e da maioria dos clones foram superiores aos $10 \%$ propostos por Green, Winandy e Kretschmann (1999), no qual o Eucalyptus grandis apresentou o menor coeficiente de variação dentre as três espécies, com 10,1\%. Aplicou-se então a ANOVA (teste $\mathrm{F}$ a $5 \%$ de significância) aos resultados, considerando-se como fonte de variação as diferentes árvores de um mesmo clone. $\mathrm{O}$ resultado do teste $\mathrm{F}$ encontra-se na Tabela 2.

TABELA 2: Teste $\mathrm{F}$ da densidade básica entre as árvores de um mesmo clone.

TABLE 2: Basic density F test between trees of a same clone.

\begin{tabular}{cccc}
\hline Clone & $\begin{array}{c}\text { Eucalyptus } \\
\text { saligna }\end{array}$ & $\begin{array}{c}\text { Eucalyptus } \\
\text { grandis }\end{array}$ & $\begin{array}{c}\text { Eucalyptus } \\
\text { dunnii }\end{array}$ \\
\hline 1 & 0,23 & 0,15 & 0,75 \\
2 & 0,15 & 0,07 & 0,07 \\
3 & 0,19 & 0,11 & 0,33 \\
4 & 0,10 & 0,27 & 0,14 \\
5 & 0,21 & 0,47 & 0,99 \\
\hline
\end{tabular}


De acordo com os resultados da Tabela 2, não houve diferença significativa ( $5 \%$ de significância) entre a densidade básica entre as árvores de um mesmo clone. Dessa maneira, a diferença obtida entre as médias de densidade básica foi intrínseca aos próprios clones. Mesmo em se tratando de clones, a heterogeneidade da madeira entre os corpos-de-prova foi responsável pelos coeficientes de variação obtidos por clone.

\section{Anisotropia de contração}

Para a discussão e definição dos clones mais instáveis dimensionalmente, tomou-se como base a contração volumétrica (Tabela 3) e o coeficiente de retratibilidade (Tabela 4) máximos, uma vez que tais valores expressam a redução total de volume da madeira na secagem. De acordo com o proposto por Rocha (2000), mesmo espécies de
Eucalyptus, reconhecidamente pouco estáveis dimensionalmente, podem apresentar coeficientes de anisotropia de contração semelhantes a espécies de elevada estabilidade dimensional, como o cedro $(1,50)$. Assim, esse coeficiente deve ser utilizado de forma criteriosa, em situações em que as demais informações de anisotropia de contração não forem disponíveis para uma determinada espécie.

$\mathrm{Na}$ Tabela 3, encontram-se os resultados do ensaio de retratibilidade.

As médias de contração máxima dos clones de Eucalyptus saligna foram inferiores àquelas obtidas por Oliveira e Silva (2003) e Malan (2000) apud Marchesan, Mattos e Shimizu (2005), porém as médias do coeficiente de anisotropia de contração foram superiores.

O clone 5 obteve a maior contração volumétrica máxima de $17,88 \%$, seguindo a tendência da densidade básica (Tabela 1). O

TABELA 3: Contrações máximas e coeficiente de anisotropia de contração para Eucalyptus saligna (S), Eucalyptus grandis (G) e Eucalyptus dunnii (D).

TABLE 3: Eucalyptus saligna (S), Eucalyptus grandis (G) and Eucalyptus dunnii (D) maximum shrinkages and anisotropic shrinkage coefficient.

\begin{tabular}{|c|c|c|c|c|c|c|c|c|c|c|c|c|}
\hline \multirow{3}{*}{ Clone } & \multicolumn{9}{|c|}{ Contração máxima (\%) } & \multirow{2}{*}{\multicolumn{3}{|c|}{$\begin{array}{c}\text { Coeficiente de } \\
\text { anisotropia }\end{array}$}} \\
\hline & \multicolumn{3}{|c|}{ Volumétrica } & \multicolumn{3}{|c|}{ Radial } & \multicolumn{3}{|c|}{ Tangencial } & & & \\
\hline & S & G & D & S & G & D & S & G & $\mathrm{D}$ & S & G & D \\
\hline 1 & $\begin{array}{c}14,52 \\
\text { B } \\
(12,8)\end{array}$ & $\begin{array}{c}13,73 \\
\text { A } \\
(9,9)\end{array}$ & $\begin{array}{c}15,57 \\
\text { A } \\
(9,1)\end{array}$ & $\begin{array}{c}4,46 \\
\text { A } \\
(13,7)\end{array}$ & $\begin{array}{c}4,74 \\
\text { BC } \\
(13,9)\end{array}$ & $\begin{array}{c}4,85 \\
\text { A } \\
(17,2)\end{array}$ & $\begin{array}{c}9,78 \\
\text { B } \\
(15,7)\end{array}$ & $\begin{array}{c}8,73 \\
\text { A } \\
(10,7)\end{array}$ & $\begin{array}{c}\text { A } \\
(5,9)\end{array}$ & $\begin{array}{c}2,32 \\
\mathrm{C} \\
(19,2)\end{array}$ & $\begin{array}{c}1,87 \\
\text { A } \\
(12,2)\end{array}$ & $\begin{array}{c}2,27 \\
\text { B } \\
(19,7)\end{array}$ \\
\hline 2 & $\begin{array}{c}16,42 \\
\text { C } \\
(14,1)\end{array}$ & $\begin{array}{c}15,31 \\
\mathrm{~B} \\
(8,8)\end{array}$ & $\begin{array}{c}19,02 \\
\text { B } \\
(19,5)\end{array}$ & $\begin{array}{c}5,21 \\
\text { B } \\
(24,7)\end{array}$ & $\begin{array}{c}4,25 \\
\text { A } \\
(10,0)\end{array}$ & $\begin{array}{c}5,30 \\
\text { A } \\
(29,5)\end{array}$ & $\begin{array}{c}10,90 \\
\text { C } \\
(11,0)\end{array}$ & $\begin{array}{c}10,80 \\
\mathrm{C} \\
(9,9)\end{array}$ & $\begin{array}{c}13,44 \\
\text { B } \\
(17,2)\end{array}$ & $\begin{array}{c}2,19 \\
\text { BC } \\
(20,0)\end{array}$ & $\begin{array}{c}2,58 \\
\text { B } \\
(16,4)\end{array}$ & $\begin{array}{c}2,67 \\
\text { C } \\
(16,7)\end{array}$ \\
\hline 3 & $\begin{array}{c}12,91 \\
\text { A } \\
(19,8)\end{array}$ & $\begin{array}{c}13,07 \\
\text { A } \\
(13,6)\end{array}$ & $\begin{array}{c}20,22 \\
\text { B } \\
(11,1)\end{array}$ & $\begin{array}{c}4,51 \\
\mathrm{AB} \\
(32,5)\end{array}$ & $\begin{array}{c}4,37 \\
\mathrm{AB} \\
(18,3)\end{array}$ & $\begin{array}{c}6,98 \\
\text { B } \\
(21,1)\end{array}$ & $\begin{array}{c}8,16 \\
\text { A } \\
(15,2)\end{array}$ & $\begin{array}{c}8,48 \\
\text { A } \\
(12,5)\end{array}$ & $\begin{array}{c}12,95 \\
\text { B } \\
(11,0)\end{array}$ & $\begin{array}{c}1,85 \\
\mathrm{~A} \\
(18,8)\end{array}$ & $\begin{array}{c}1,95 \\
\mathrm{~A} \\
(10,3)\end{array}$ & $\begin{array}{c}1,90 \\
\text { A } \\
(13,7)\end{array}$ \\
\hline 4 & $\begin{array}{c}14,18 \\
\text { B } \\
(19,5)\end{array}$ & $\begin{array}{c}13,57 \\
\text { A } \\
(9,9)\end{array}$ & $\begin{array}{c}19,79 \\
\text { B } \\
(22,2)\end{array}$ & $\begin{array}{c}4,64 \\
\mathrm{AB} \\
(27,1)\end{array}$ & $\begin{array}{c}4,59 \\
\mathrm{AB} \\
(14,1)\end{array}$ & $\begin{array}{c}6,21 \\
\mathrm{~B} \\
(35,1)\end{array}$ & $\begin{array}{c}9,28 \\
\text { B } \\
(16,2)\end{array}$ & $\begin{array}{c}8,75 \\
\text { A } \\
(10,3)\end{array}$ & $\begin{array}{c}13,31 \\
\text { B } \\
(18,4)\end{array}$ & $\begin{array}{c}2,09 \\
\text { B } \\
(20,9)\end{array}$ & $\begin{array}{c}1,93 \\
\mathrm{~A} \\
(14,1)\end{array}$ & $\begin{array}{c}2,25 \\
\text { B } \\
(19,9)\end{array}$ \\
\hline 5 & $\begin{array}{c}17,88 \\
\text { D } \\
(14,9)\end{array}$ & $\begin{array}{c}14,81 \\
\text { B } \\
(9,9)\end{array}$ & $\begin{array}{c}18,75 \\
\text { B } \\
(17,1)\end{array}$ & $\begin{array}{c}6,37 \\
\mathrm{C} \\
(26,6)\end{array}$ & $\begin{array}{c}5,03 \\
\mathrm{C} \\
(18,6)\end{array}$ & $\begin{array}{c}5,12 \\
\text { A } \\
(26,2)\end{array}$ & $\begin{array}{c}11,24 \\
\mathrm{C} \\
(11,3)\end{array}$ & $\begin{array}{c}9,52 \\
\mathrm{~B} \\
(7,6)\end{array}$ & $\begin{array}{c}13,35 \\
\mathrm{~B} \\
(15,0)\end{array}$ & $\begin{array}{c}1,83 \\
\text { A } \\
(21,5)\end{array}$ & $\begin{array}{c}1,93 \\
\text { A } \\
(14,4)\end{array}$ & $\begin{array}{c}2,71 \\
\text { C } \\
(20,2)\end{array}$ \\
\hline Espécie & $\begin{array}{l}15,16 \mathrm{~b} \\
(16,2)\end{array}$ & $\begin{array}{c}14,10 \mathrm{a} \\
(4,6)\end{array}$ & $\begin{array}{c}18,71 \mathrm{c} \\
(15,8)\end{array}$ & $\begin{array}{l}5,03 \mathrm{~b} \\
(24,9)\end{array}$ & $\begin{array}{l}4,60 \mathrm{a} \\
(15,0)\end{array}$ & $\begin{array}{l}5,70 \mathrm{c} \\
(25,8)\end{array}$ & $\begin{array}{l}9,86 \mathrm{~b} \\
(13,9)\end{array}$ & $\begin{array}{l}9,25 \mathrm{a} \\
(10,2)\end{array}$ & $\begin{array}{l}12,74 \mathrm{c} \\
(13,5)\end{array}$ & $\begin{array}{l}2,06 a \\
(19,9)\end{array}$ & $\begin{array}{l}2,05 \mathrm{a} \\
(13,5)\end{array}$ & $\begin{array}{l}2,36 \mathrm{~b} \\
(22,6)\end{array}$ \\
\hline
\end{tabular}

Médias seguidas por uma mesma letra maiúscula em uma mesma coluna e por uma mesma letra minúscula em uma mesma linha não diferem entre si de acordo com o teste de Tukey a 5\% de significância. Resultados entre parênteses correspondem ao coeficiente de variação (\%).

Ci. Fl., v. 20, n. 4, out.-dez., 2010 
coeficiente de correlação de Pearson (r) entre a densidade básica e a contração volumétrica máxima foi de 0,89 , indicando a associação positiva entre tais variáveis. O clone 3 obteve a menor média de contração volumétrica máxima, acompanhando $\mathrm{o}$ resultado de densidade básica. $\mathrm{O}$ coeficiente $\mathrm{r}$ entre a densidade básica e a contração volumétrica máxima do clone 3 foi de 0,70 . Os clones 1,3 e 4 obtiveram coeficiente $\mathrm{r}$ respectivos de $0,86,0,76 \mathrm{e}$ 0,71 , indicando que a associação entre essas duas variáveis para os clones de Eucalyptus saligna foi positiva e diretamente proporcional.

As médias de contração máxima dos clones de Eucalyptus grandis foram inferiores àquelas obtidas por Gonçalez et al. (2006) e Silva et al. (2006), no entanto, as médias do coeficiente de anisotropia de contração foram superiores.

O clone 2 obteve a maior média absoluta de contração volumétrica máxima, com 15,31\%, e obteve a maior média de densidade básica (Tabela 1). Porém, a contração volumétrica máxima do clone 2 não diferiu estatisticamente do clone 5 , com média de $14,81 \%$. O coeficiente $r$ entre a densidade básica e a contração volumétrica máxima do clone 2 foi de 0,89 , enquanto do clone 5 foi de 0,60, indicando um maior grau de associação entre essas variáveis para o clone 2 .

O clone 3 obteve a menor média absoluta de contração volumétrica máxima, com 13,07\%, e obteve a menor média de densidade básica (Tabela 1). Porém, a contração volumétrica máxima do clone 3 não diferiu estatisticamente dos clones $4 \mathrm{e}$ 1, com, respectivamente, 13,57 e $13,73 \%$. Mesmo para as médias do coeficiente de anisotropia de contração não houve diferença significativa entre os três clones. O baixo coeficiente de variação da contração volumétrica máxima dos três clones sugere homogeneidade entre as propriedades de diferentes árvores de um mesmo clone e minimiza a possibilidade de erros de amostragem para explicar a não diferenciação significativa entre eles.

Sugere-se que os clones 1 e 4 tenham teor de extrativos significativamente mais elevados que o clone 3 , uma vez que aqueles obtiveram densidade básica superior a este, porém com contração volumétrica estatisticamente não diferente. Essa pressuposição embasou-se em Tsoumis (1991), que afirmou que a redução da contração e do inchamento da madeira é proporcional à quantidade de extrativos presentes na parede celular. Porém, essa suposição é passiva de investigação futura para a sua confirmação.
Os coeficientes $r$ entre a densidade básica e a contração volumétrica máxima dos clones 1 , 3 e 4 foram, respectivamente, $0,70,0,70$ e 0,72 , demonstrando associação positiva e diretamente proporcional entre tais variáveis. Os clones 2 e 5 obtiveram coeficiente $\mathrm{r}$ de 0,60 , denotando um menor grau de associação entre as duas variáveis.

As médias de contração máxima dos clones de Eucalyptus dunnii foram inferiores àquelas obtidas por Severo (1998) e Rocha (2000), no entanto, as médias do coeficiente de anisotropia de contração foram superiores.

O clone 3 obteve a maior média absoluta de contração volumétrica máxima, com 20,22\%, e a maior densidade básica (Tabela 1), porém não diferiu estatisticamente dos clones 4, 2 e 5 com, respectivamente, $19,79,19,02$ e $18,75 \%$. Dentre estes, o clone 5 obteve a maior média absoluta de coeficiente de anisotropia de contração, com 2,71, porém não diferiu estatisticamente do clone 2 , com 2,67 . Os clones $2,3,4$ e 5 obtiveram coeficientes $r$ de $0,88,0,76,0,75$ e 0,74 respectivamente, denotando associação positiva e diretamente proporcional entre densidade básica e contração volumétrica máxima.

O clone 1 obteve a menor contração volumétrica máxima, com 15,57\%, seguindo o resultado de densidade básica (Tabela 1). O coeficiente $r$ entre a densidade básica e a contração máxima desse clone foi de 0,78 , denotando, à semelhança dos demais clones dessa espécie, que essas variáveis se relacionam diretamente.

Igualmente à densidade básica, aplicouse a ANOVA (teste $\mathrm{F}$ a 5\% de significância) aos resultados, considerando-se como fonte de variação as diferentes árvores de um mesmo clone. $\mathrm{O}$ resultado do teste $\mathrm{F}$ encontra-se na Tabela 4.

TABELA 4: Teste $F$ da contração volumétrica máxima entre as árvores de um mesmo clone.

TABLE 4: Maximum volumetric shrinkage $F$ test between trees of a same clone.

\begin{tabular}{cccc}
\hline Clone & $\begin{array}{c}\text { Eucalyptus } \\
\text { saligna }\end{array}$ & $\begin{array}{c}\text { Eucalyptus } \\
\text { grandis }\end{array}$ & $\begin{array}{c}\text { Eucalyptus } \\
\text { dunnii }\end{array}$ \\
\hline 1 & 0,34 & 3,83 & 0,67 \\
2 & 1,26 & 1,95 & 1,78 \\
3 & 2,79 & 1,78 & 2,63 \\
4 & 0,53 & 0,36 & 0,46 \\
5 & 0,47 & 1,69 & 1,03 \\
\hline
\end{tabular}


De acordo com os resultados da Tabela 4, não houve diferença significativa ( $5 \%$ de significância) entre a contração volumétrica máxima entre as árvores de um mesmo clone, o mesmo resultado obtido para a densidade básica.

$\mathrm{Na}$ Tabela 5, encontram-se os resultados de coeficiente de retratibilidade.

O coeficiente de retratibilidade expressa a variação dimensional (em \%) para cada $1 \%$ de variação de umidade. Como na prática, a madeira não é seca a $0 \%$ de umidade, o conhecimento do coeficiente retratibilidade torna-se importante, em relação às contrações máximas, para o cálculo da redução de volume da madeira por contração de um determinado teor superior de umidade até outro inferior.

O clone 5 de Eucalyptus saligna apresentou o maior coeficiente de retratibilidade máximo, com $0,58 \% / \%$. Esse resultado seguiu a mesma tendência daqueles de contração volumétrica máxima. Os demais clones não diferiram estatisticamente entre si.

O clone 5 de Eucalyptus grandis obteve o maior coeficiente de retratibilidade absoluto, de $0,48 \% / \%$, porém não diferiu estatisticamente dos clones 1 e 2 , ambos com $0,46 \%$. O clone 3 obteve a menor média absoluta, de $0,41 \%$, porém não diferiu estatisticamente do clone 4 , com $0,44 \%$.

O clone 3 de Eucalyptus dunnii obteve o maior coeficiente de retratibilidade volumétrico, de $0,68 \%$. O clone 1 obteve a menor média absoluta, de $0,49 \%$, porém não diferiu estatisticamente dos clones 2 e 5 com, respectivamente, 0,52 e $0,51 \%$.

\section{Comparação entre espécies}

Analisando-se entre espécies, o Eucalyptus dunnii obteve as maiores médias de anisotropia de contração e também a maior densidade básica.

A amplitude da densidade básica foi de

TABELA 5: Coeficiente de retratibilidade para clones de Eucalyptus saligna (S), Eucalyptus grandis (G) e Eucalyptus dunnii (D).

TABLE 5: Shrinkage coefficient of Eucalyptus saligna (S), Eucalyptus grandis (G) and Eucalyptus dunnii (D) clones.

\begin{tabular}{|c|c|c|c|c|c|c|c|c|c|}
\hline \multirow{3}{*}{ Clone } & \multicolumn{9}{|c|}{ Coeficiente de retratibilidade máximo (\%/\%) } \\
\hline & \multicolumn{3}{|c|}{ Volumétrico } & \multicolumn{3}{|c|}{ Radial } & \multicolumn{3}{|c|}{ Tangencial } \\
\hline & S & G & $\mathrm{D}$ & S & G & $\mathrm{D}$ & S & G & $\mathrm{D}$ \\
\hline 1 & $\begin{array}{c}0,49 \\
\text { A } \\
(19,2)\end{array}$ & $\begin{array}{c}0,46 \\
\mathrm{BC} \\
(11,9)\end{array}$ & $\begin{array}{c}0,49 \\
\text { A } \\
(30,0)\end{array}$ & $\begin{array}{c}0,19 \\
\text { A } \\
(30,5)\end{array}$ & $\begin{array}{c}0,19 \\
\text { B } \\
(14,1)\end{array}$ & $\begin{array}{c}0,19 \\
\text { A } \\
(36,8)\end{array}$ & $\begin{array}{c}0,30 \\
\text { B } \\
(14,1)\end{array}$ & $\begin{array}{c}0,27 \\
\mathrm{BC} \\
(13,3)\end{array}$ & $\begin{array}{c}0,30 \\
\text { A } \\
(29,9)\end{array}$ \\
\hline 2 & $\begin{array}{c}0,50 \\
\mathrm{~A} \\
(16,0)\end{array}$ & $\begin{array}{c}0,46 \\
\text { BC } \\
(13,5)\end{array}$ & $\begin{array}{c}0,52 \\
\text { A } \\
(17,8)\end{array}$ & $\begin{array}{c}0,20 \\
\text { A } \\
(24,5)\end{array}$ & $\begin{array}{c}0,17 \\
\text { A } \\
(11,9)\end{array}$ & $\begin{array}{c}0,20 \\
\text { A } \\
(30,0)\end{array}$ & $\begin{array}{c}0,28 \\
\mathrm{AB} \\
(13,6)\end{array}$ & $\begin{array}{c}0,29 \\
\mathrm{C} \\
(18,3)\end{array}$ & $\begin{array}{c}0,31 \\
\text { A } \\
(16,9)\end{array}$ \\
\hline 3 & $\begin{array}{c}0,48 \\
\text { A } \\
(9,2)\end{array}$ & $\begin{array}{c}0,41 \\
\text { A } \\
(11,9)\end{array}$ & $\begin{array}{c}0,68 \\
\mathrm{C} \\
(11,4)\end{array}$ & $\begin{array}{c}0,19 \\
\text { A } \\
(13,1)\end{array}$ & $\begin{array}{c}0,16 \\
\text { A } \\
(15,6)\end{array}$ & $\begin{array}{c}0,29 \\
\mathrm{C} \\
(16,1)\end{array}$ & $\begin{array}{c}0,28 \\
\mathrm{AB} \\
(12,9)\end{array}$ & $\begin{array}{c}0,24 \\
\text { A } \\
(13,7)\end{array}$ & $\begin{array}{c}0,39 \\
\text { C } \\
(12,6)\end{array}$ \\
\hline 4 & $\begin{array}{c}0,47 \\
\text { A } \\
(13,8)\end{array}$ & $\begin{array}{c}0,44 \\
\mathrm{AB} \\
(7,5)\end{array}$ & $\begin{array}{c}0,60 \\
\text { B } \\
(14,6)\end{array}$ & $\begin{array}{c}0,19 \\
\text { A } \\
(22,6)\end{array}$ & $\begin{array}{c}0,17 \\
\text { A } \\
(12,3)\end{array}$ & $\begin{array}{c}0,24 \\
\mathrm{~B} \\
(23,7)\end{array}$ & $\begin{array}{c}0,27 \\
\mathrm{~A} \\
(12,8)\end{array}$ & $\begin{array}{c}0,26 \\
\mathrm{AB} \\
(8,1)\end{array}$ & $\begin{array}{c}0,35 \\
\mathrm{~B} \\
(11,7)\end{array}$ \\
\hline 5 & $\begin{array}{c}0,58 \\
\text { B } \\
(19,5)\end{array}$ & $\begin{array}{c}0,48 \\
\mathrm{C} \\
(11,4)\end{array}$ & $\begin{array}{c}0,51 \\
\mathrm{~A} \\
(15,3)\end{array}$ & $\begin{array}{c}0,24 \\
\text { B } \\
(24,8)\end{array}$ & $\begin{array}{c}0,19 \\
\text { B } \\
(15,3)\end{array}$ & $\begin{array}{c}0,20 \\
\mathrm{~A} \\
(18,9)\end{array}$ & $\begin{array}{c}0,33 \\
\text { C } \\
(19,)\end{array}$ & $\begin{array}{c}0,27 \\
\text { BC } \\
(11,7)\end{array}$ & $\begin{array}{c}0,31 \\
\text { A } \\
(16,5)\end{array}$ \\
\hline Espécie & $\begin{array}{l}0,50 b \\
(15,4)\end{array}$ & $\begin{array}{c}0,45 a \\
(12,5)\end{array}$ & $\begin{array}{c}0,56 \mathrm{c} \\
(21,5)\end{array}$ & $\begin{array}{c}0,20 \mathrm{~b} \\
(23,1)\end{array}$ & $\begin{array}{c}0,18 \mathrm{a} \\
(15,4)\end{array}$ & $\begin{array}{c}0,23 \mathrm{c} \\
(29,1)\end{array}$ & $\begin{array}{l}0,29 b \\
(14,5)\end{array}$ & $\begin{array}{c}0,26 a \\
(15,1)\end{array}$ & $\begin{array}{c}0,33 \mathrm{c} \\
(20,5)\end{array}$ \\
\hline
\end{tabular}

Médias seguidas por uma mesma letra maiúscula em uma mesma coluna por uma mesma letra minúscula em uma mesma linha não diferem entre si de acordo com o teste de Tukey a 5\% de significância. Resultados entre parênteses correspondem ao coeficiente de variação (\%).

Ci. Fl., v. 20, n. 4, out.-dez., 2010 
$0,14 \mathrm{~g} / \mathrm{cm}^{3}$ para os clones de Eucalyptus saligna, que variaram de $0,42 \mathrm{~g} / \mathrm{cm}^{3}$ a $0,56 \mathrm{~g} / \mathrm{cm}^{3}$ e também para os clones de Eucalyptus dunnii, que variaram de $0,51 \mathrm{~g} / \mathrm{cm}^{3}$ a $0,65 \mathrm{~g} / \mathrm{cm}^{3}$. Os clones de Eucalyptus grandis foram os mais homogêneos, variando $0,09 \mathrm{~g} /$ $\mathrm{cm}^{3}$ ou $90 \mathrm{~kg} / \mathrm{m}^{3}\left(0,40 \mathrm{~g} / \mathrm{cm}^{3}-0,49 \mathrm{~g} / \mathrm{cm}^{3}\right)$, e também obtiveram o menor coeficiente de variação, sendo a espécie mais recomendada onde a homogeneidade da densidade básica seja requerida, dentre os clones estudados.

Para as espécies, ficou clara a relação de quanto maior a densidade básica maior a contração volumétrica máxima. $\mathrm{O}$ coeficiente de correlação de Pearson entre essas duas variáveis foi de 0,84 , 0,72 e 0,73, respectivamente, para Eucalyptus saligna, Eucalyptus grandis e Eucalyptus dunnii, comprovando uma relação positiva e diretamente proporcional.

O Eucalyptus grandis foi a espécie mais estável dimensionalmente, com as menores médias de contração máxima (volumétrica - 14,10\%; radial $-4,60 \%$; tangencial $-9,25 \%)$ e de coeficiente de retratibilidade (volumétrico - 0,45\%/\%; radial - 0,18\%/\%; tangencial - 0,26\%/\%), e somente para o coeficiente de anisotropia de contração não houve diferença significativa entre as médias de Eucalyptus grandis e Eucalyptus saligna, com 2,05 e 2,06 respectivamente. Todas as espécies deste trabalho, de acordo com o critério do coeficiente de anisotropia de contração estabelecido por Klitzke (2007), foram classificadas como de média instabilidade dimensional, ou seja, entre 2,0 e 2,5.

O Eucalyptus dunnii foi a espécie mais instável dimensionalmente, com as maiores médias de contração máxima (volumétrica - 18,71\%; radial $-5,70 \%$; tangencial $-12,74 \%$ ) e de coeficiente de retratibilidade (volumétrico - 0,56\%/\%; radial $0,23 \% / \%$; tangencial - 0,33\%/\%). O Eucalyptus saligna apresentou resultados intermediários, porém semelhantes às médias apresentadas pelo Eucalyptus grandis.

As informações das propriedades físicas das madeiras dos clones podem auxiliar na adequada indicação da aplicação final, uma vez que a densidade básica se correlaciona com resistência mecânica, porosidade, facilidade de secagem e penetração de preservantes, poder calorífico, acabamentos externos, trabalhabilidade, laminação, rendimento de polpação e fator de compactação de painéis aglomerados. Além disso, o conhecimento da estabilidade dimensional desses clones pode ser utilizado como subsídio para a limitação ou aceitação das madeiras onde tal propriedade seja requerida.

Cuidado especial deve ser tomado no melhoramento genético dos clones, por meio do qual a silvicultura frequentemente busca aumentar o incremento volumétrico, uma vez que a velocidade de crescimento influencia nas propriedades físicas da madeira dos clones de Eucalyptus (NUTTO, 2007).

Assim, tais informações proporcionam a utilização mais racional da madeira dos 15 clones do gênero Eucalyptus.

\section{CONCLUSÕES}

Houve relação direta entre densidade básica, contração volumétrica máxima e coeficiente de retratibilidade volumétrico máximo entre as espécies.

O clone 5 de Eucalyptus saligna obteve a maior densidade básica $\left(0,56 \mathrm{~g} / \mathrm{cm}^{3}\right)$ e foi o mais instável dimensionalmente apresentando ainda relação direta entre densidade básica e coeficiente de retratibilidade volumétrico máximo. Considerandose como critério a contração volumétrica máxima, o clone 3 foi o mais estável dimensionalmente.

Para o Eucalyptus grandis, os clones 2 e 3 obtiveram respectivamente a menor e maior densidade básica, com 0,40 e $0,49 \mathrm{~g} / \mathrm{cm}^{3}$. Não foi possível distinguir dentre os clones 1, 3 e 4 qual foi o mais estável dimensionalmente e, considerandose como critério o coeficiente de retratibilidade volumétrico máximo, o clone 5 foi o mais instável dimensionalmente.

Para as espécies Eucalyptus saligna e Eucalyptus dunnii, não foi possível concluir qual clone obteve a menor densidade básica.

O clone 3 de Eucalyptus dunnii obteve a maior densidade básica $\left(0,65 \mathrm{~g} / \mathrm{cm}^{3}\right) \mathrm{e}$, considerandose como critério o coeficiente de retratibilidade volumétrico máximo, foi o clone mais instável dimensionalmente. Considerando-se a contração volumétrica máxima, o clone 1 foi o mais estável dimensionalmente.

Esperava-se uma relação direta entre a contração volumétrica máxima e o coeficiente de retratibilidade volumétrico máximo, porém não houve um padrão definido entre os clones.

Observou-se homogeneidade entre as propriedades de densidade básica e contração volumétrica máxima entre as árvores de um mesmo clone. 
O Eucalyptus grandis foi a espécie de menor densidade e maior estabilidade dimensional, enquanto o Eucalyptus dunnii foi a espécie de maior densidade e maior instabilidade dimensional.

\section{REFERÊNCIAS BIBLIOGRÁFICAS}

ANDRADE, A.; JANKOWSKY, I. P.; DUCATTI, M. A. Grupamento de madeiras para secagem convencional. Scientia Forestalis, Piracicaba, n. 59, p. 89-99, jun. 2001.

CINIGLIO, G. Avaliação da secagem de madeira serrada de E.grandis e E.urophylla. 1998. 73 f. Dissertação (Mestrado)-Escola Superior de Agricultura Luiz de Queiroz/USP, Piracicab, 1998. COMISIÓN Panamericana de Normas Técnicas. Maderas: Método de determinación del peso específico aparente. Buenos Aires: COPANT, 1972. $461.5 \mathrm{p}$.

COMISIÓN Panamericana de Normas Técnicas. Maderas: Método de determinación de la contracción. Buenos Aires: COPANT, 1972. 462. 5 p.

GONÇALEZ, J. C. et al. Características Tecnológicas das madeiras de Eucalyptus grandis W.Hill ex Maiden e Eucalyptus cloeziana F. Muell visando ao seu aproveitamento na indústria moveleira. Ciência Florestal, Santa Maria, v. 16, n. 3, p. 329-341. 2006.

GREEN,D.W.;WINANDY,J.E;KRETSCHMANN, D. E. Mechanical properties of wood. In: FPL. Wood handbook: wood as an engineering material. Madison: United States Department of Agriculture, 1999. Capítulo 4.

IPT-Instituto de Pesquisas Tecnológicas. Disponível em: $<$ ( http://www.ipt.br/informacoes_madeiras3. php? madeira $=13$ ) $>$ Acesso em: 1 de outubro de 2009 . KLITZKE, R. J. Secagem da Madeira. In: OLIVEIRA, J. T. S. et al. Tecnologias Aplicadas ao Setor Madeireiro. Jerônimo Monteiro: Suprema, 2007. p. 271-342.

KOLLMANN, F. F. P.; CÔTÉ JUNIOR, W. A. Principles of Wood Science and Technology. Berlin: Springer-Verlag, 1968. 592 p.

MARCHESAN, R., MATTOS, P. P., SHIMIZU, J. Y. Caracterização física, química e anatômica da madeira de Eucalyptus microcorys F. Muell. Colombo: Embrapa Florestas, 2005. 5 p.(Comunicado Técnico 133)

MELO, J. E. Madeira: características e aplicações. Brasília: LPF, 2002. 30 p.

NUTTO, L. Propriedades da madeira: qualidade da madeira em florestas plantadas. In: Palestra proferida em aula teórica da disciplina AT 702 (Propriedades da Madeira) no programa de pósgraduação em Engenharia Florestal da Universidade Federal do Paraná. 2007. Curitiba, 60 p.

OLIVEIRA, J. T. SILVA, J. C. Variação radial da retratibilidade e densidade básica da madeira de Eucalyptus saligna Sm. Revista Árvore, Viçosa, v. 27, n. 3, p. 381-385, 2003.

PEREIRA, J. C. D. et al. Características de madeiras de algumas espécies de eucaliptos plantadas no Brasil. Colombo; Embrapa Florestas, 2000. 113 p. (Embrapa Florestas. Documentos, 38). ROCHA, M. P. Eucalyptus grandis Hill ex Maiden e Eucalyptus dunnii Maiden como fontes de matéria-prima para Serrarias. 2000. 185 f. Tese (Doutorado)-Universidade Federal do Paraná, Curitiba, 2000.

SEVERO, E. T. D. Estudo sobre o efeito da vaporização nas propriedades e comportamento de secagem da madeira de Eucalyptus dunnii Maid. 1998. 200 f. Tese (Doutorado)-Universidade Federal do Paraná, Curitiba1998.

SILVA, J. C. et al. Variação da retratibilidade da madeira de Eucalyptus grandis Hill ex Maiden, em função da idade e da posição radial no tronco. Revista Árvore, Viçosa, v. 30, n. 5, p. 803-810, 2006.

TOMAZELLO FILHO, M. Variação radial da densidade básica e da estrutura anatômica de Eucalyptus saligna e Eucalyptus grandis. IPEF, Piracicaba, n. 29, p. 37-45, abr.1985

TRUGILHO, P. F., LIMA, J. T., MENDES, L. M. Influência da idade nas características físicoquímicas e anatômicas da madeira de Eucalyptus saligna. Cerne, Lavras, v. 2, n. 1, 1996.

TRUGILHO, P.F. et al. Deformação residual longitudinal (DRL) e tangencial (DRT) em seis clones de Eucalyptus spp. Cerne, Lavras, v. 12, n. 3, p. 279-286, jul/set, 2006. 